\title{
Elastic scattering and breakup reactions with light proton- and neutron-rich exotic nuclei
}

\author{
M. K. Gaidarov ${ }^{1, *}, V . K$. Lukyanov $^{2}, D . N . \mathrm{Kadrev}^{1}, E . V . Z_{\text {Zemlyanaya }}^{2}, A . N$. Antonov $^{1}, K . V$. Lukyanov $^{2}$, and \\ K. Spasova ${ }^{1}$ \\ ${ }^{1}$ Institute for Nuclear Research and Nuclear Energy, Bulgarian Academy of Sciences, Sofia 1784, Bulgaria \\ ${ }^{2}$ Joint Institute for Nuclear Research, Dubna 141980, Russia
}

\begin{abstract}
A microscopic analysis of the optical potentials (OPs) and cross sections of elastic scattering of ${ }^{8} \mathrm{~B}$ on ${ }^{12} \mathrm{C},{ }^{58} \mathrm{Ni}$, and ${ }^{208} \mathrm{~Pb}$ targets at energies $20<E<170 \mathrm{MeV}$ and ${ }^{12,14} \mathrm{Be}$ on ${ }^{12} \mathrm{C}$ at $56 \mathrm{MeV} /$ nucleon is carried out. The real part of the OP is calculated by a folding procedure and the imaginary part is obtained on the base of the high-energy approximation (HEA). The density distributions of ${ }^{8} \mathrm{~B}$ evaluated within the variational Monte Carlo (VMC) model and the three-cluster model (3CM) are used to construct the potentials. The ${ }^{14} \mathrm{Be}$ densities obtained in the framework of the the generator coordinate method (GCM) are used to calculate the optical potentials, while for the same purpose both the VMC model and GCM densities of ${ }^{12} \mathrm{Be}$ are used. In the hybrid model developed and explored in our previous works, the only free parameters are the depths of the real and imaginary parts of OP obtained by fitting the experimental data. The use of HEA to estimate the imaginary $\mathrm{OP}$ at energies just above the Coulomb barrier is discussed. In addition, cluster model, in which ${ }^{8} \mathrm{~B}$ consists of a $p$-halo and the ${ }^{7} \mathrm{Be}$ core, is applied to calculate the breakup cross sections of ${ }^{8} \mathrm{~B}$ nucleus on ${ }^{9} \mathrm{Be},{ }^{12} \mathrm{C}$, and ${ }^{197} \mathrm{Au}$ targets, as well as momentum distributions of ${ }^{7} \mathrm{Be}$ fragments. A good agreement of the theoretical results with the available experimental data is obtained. It is concluded that the reaction studies performed in this work may provide supplemental information on the internal spatial structure of the proton- and neutron-halo nuclei.
\end{abstract}

\section{Introduction}

Recently, research on short-lived unstable nuclei has become very active following the discovery of exotic phenomena, such as neutron halo, neutron skin, and the disappearance of the magic numbers. Although less probable, proton halos are also possible. In recent years, the short-lived radioactive nucleus ${ }^{8} \mathrm{~B}$ with a very low breakup threshold energy $(0.137 \mathrm{MeV})$, adjacent to the proton drip line, has attracted much attention because it may present a proton halo structure and is valuable for astrophysical reasons $[1,2]$. The narrow momentum distributions of ${ }^{7} \mathrm{Be}$ fragments in the breakup of ${ }^{8} \mathrm{~B}$ measured in $\mathrm{C}, \mathrm{Al}$, and $\mathrm{Pb}$ targets at $1471 \mathrm{MeV} /$ nucleon with full width at half maximum (FWHM) of $81 \pm 6 \mathrm{MeV} / \mathrm{c}$ in all targets have been interpreted in terms of a largely extended proton distribution for ${ }^{8} \mathrm{~B}$ and have implied a radius of $2.78 \mathrm{fm}$ [3]. Here we should mention also the results of the experiments at lower energies for the breakup of ${ }^{8} \mathrm{~B}$ in the collisions with $\mathrm{Be}$ and $\mathrm{Au}$ targets at $41 \mathrm{MeV} /$ nucleon $(81 \pm 4$ and $62 \pm 3$ $\mathrm{MeV} / \mathrm{c} \mathrm{FWHM}$ for Be and Au targets, respectively) [4] and for $\mathrm{C}$ target at $36 \mathrm{MeV} /$ nucleon [5] with FWHM $124 \pm 17$ and $92 \pm 7 \mathrm{MeV} / \mathrm{c}$ for the stripping and diffraction components, correspondingly. Indeed, these experimental results reflect the large spatial extension of the loosely bound proton in ${ }^{8} \mathrm{~B}$. The halo nature of ${ }^{8} \mathrm{~B}$ nucleus through studies of its breakup has been mostly tested with cluster mod-

\footnotetext{
*e-mail: gaidarov@inrne.bas.bg
}

els presuming simple two-cluster structure that consists of ${ }^{7} \mathrm{Be}$ core and valence proton (for instance, Refs. [3, 6]).

The present work (see also [7]) aims first, to analyze the differential elastic cross sections for the scattering of ${ }^{8} \mathrm{~B}$ on ${ }^{12} \mathrm{C}$ at $25.8 \mathrm{MeV}[8],{ }^{8} \mathrm{~B}$ on ${ }^{58} \mathrm{Ni}$ at $20.7,23.4,25.3$, 27.2, and $29.3 \mathrm{MeV}$ [9], and ${ }^{8} \mathrm{~B}$ on ${ }^{208} \mathrm{~Pb}$ at $170.3 \mathrm{MeV}$ [10] within the microscopic model of the respective OP and compare the results with the available experimental data. The same model is applied to calculate the elastic scattering ${ }^{12,14} \mathrm{Be}+{ }^{12} \mathrm{C}$ cross sections at $56 \mathrm{MeV} /$ nucleon. Such a study could lead to a minimization of the ambiguities in the fitted OPs. As in our previous works [11-15], where processes with neutron-rich $\mathrm{He}, \mathrm{Li}$, and $\mathrm{Be}$ isotopes were considered, we use the hybrid model of OP [16], in which the real part (ReOP) is calculated by a folding of a nuclear density and the effective nucleon-nucleon (NN) potentials [17] including direct and exchange isoscalar (IS) and isovector (IV) parts. The imaginary part (ImOP) is obtained on the base of the high-energy approximation method developed in Refs. [18, 19]. There are only two fitting parameters in the hybrid model, which are related to the depths of the ReOP and ImOP. Second, for a more consistent description of the possible halo structure of ${ }^{8} \mathrm{~B}$ we calculate the momentum distributions of ${ }^{7} \mathrm{Be}$ fragments from the breakup reactions ${ }^{8} \mathrm{~B}+{ }^{9} \mathrm{Be},{ }^{8} \mathrm{~B}+{ }^{12} \mathrm{C}$, and ${ }^{8} \mathrm{~B}+{ }^{197} \mathrm{Au}$ for which experimental data are available. Such a complex study based on the microscopic method to obtain the OPs with a minimal number of free parame- 
ters and by testing density distributions of ${ }^{8} \mathrm{~B}$ which reflect its proton-halo structure (in contrast, e.g., to the HartreeFock density used in Ref. [10]) would lead to a better understanding of the ${ }^{8} \mathrm{~B}$ structure and to a reduction of the inconsistency of describing the available data.

\section{Elastic scattering of ${ }^{8} \mathrm{~B}$ on ${ }^{12} \mathrm{C},{ }^{58} \mathrm{Ni}$, and ${ }^{208} \mathrm{~Pb}$ and ${ }^{12,14} \mathrm{Be}$ on ${ }^{12} \mathrm{C}$ targets}

The microscopic volume OP used in our calculations contains the real part $\left(V^{D F}\right)$ including both the direct and exchange terms and the HEA microscopically calculated imaginary part $\left(W^{H}\right)$. It has the form

$$
U(r)=N_{R} V^{D F}(r)+i N_{I} W^{H}(r) .
$$

The parameters $N_{R}$ and $N_{I}$ entering Eq. (1) renormalize the strength of OP and are fitted by comparison with the experimental cross sections. The real part $V^{D F}$ consists of the direct $\left(V^{D}\right)$ and exchange $\left(V^{E X}\right)$ double-folding integrals that include effective NN potentials and density distribution functions of colliding nuclei. The $V^{D}$ and $V^{E X}$ parts of the ReOP have isoscalar (IS) and isovector (IV) contributions. The IS ones of both terms are:

$$
\begin{aligned}
V_{I S}^{D}(r)= & \int d^{3} r_{p} d^{3} r_{t} \rho_{p}\left(\vec{r}_{p}\right) \rho_{t}\left(\vec{r}_{t}\right) v_{N N}^{D}(s), \\
V_{I S}^{E X}(r)= & \int d^{3} r_{p} d^{3} r_{t} \rho_{p}\left(\vec{r}_{p}, \vec{r}_{p}+\vec{s}\right) \rho_{t}\left(\vec{r}_{t}, \vec{r}_{t}-\vec{s}\right) \\
& \times v_{N N}^{E X}(s) \exp \left[\frac{i \vec{K}(r) \cdot s}{M}\right],
\end{aligned}
$$

where $\vec{s}=\vec{r}+\vec{r}_{t}-\vec{r}_{p}$ is the vector between two nucleons, one of which belongs to the projectile and another one to the target nucleus. In Eq. (2), $\rho_{p}\left(\vec{r}_{p}\right)$ and $\rho_{t}\left(\vec{r}_{t}\right)$ are the densities of the projectile and the target, respectively, while in Eq. (3) $\rho_{p}\left(\vec{r}_{p}, \vec{r}_{p}+\vec{s}\right)$ and $\rho_{t}\left(\vec{r}_{t}, \vec{r}_{t}-\vec{s}\right)$ are the density matrices for the projectile and the target that are usually taken in an approximate form. The effective NN interactions $v_{N N}^{D}$ and $v_{N N}^{E X}$ have their IS and IV components in the form of M3Y interaction obtained within $g$-matrix calculations using the Paris NN potential [17]. The expressions for the energy and density dependence of the effective NN interaction are given, e.g., in Ref. [15].

Concerning the ImOP, it corresponds to the full microscopic OP derived in Refs. [16, 20] within the HEA $[18,19]$

$$
W^{H}(r)=-\frac{1}{2 \pi^{2}} \frac{E}{k} \bar{\sigma}_{N} \int_{0}^{\infty} j_{0}(q r) \rho_{p}(q) \rho_{t}(q) f_{N}(q) q^{2} d q .
$$

In Eq. (4), $\rho(q)$ are the corresponding form factors of the nuclear densities, $f_{N}(q)$ is the amplitude of the NN scattering and $\bar{\sigma}_{N}$ is the averaged over the isospin of the nucleus total NN scattering cross section, both accounting for the in-medium effects.

In the present work we use the density distribution of ${ }^{8} \mathrm{~B}$ nucleus obtained within the variational Monte Carlo model [21] and also the density obtained within the framework of the microscopic three-cluster model of Varga et
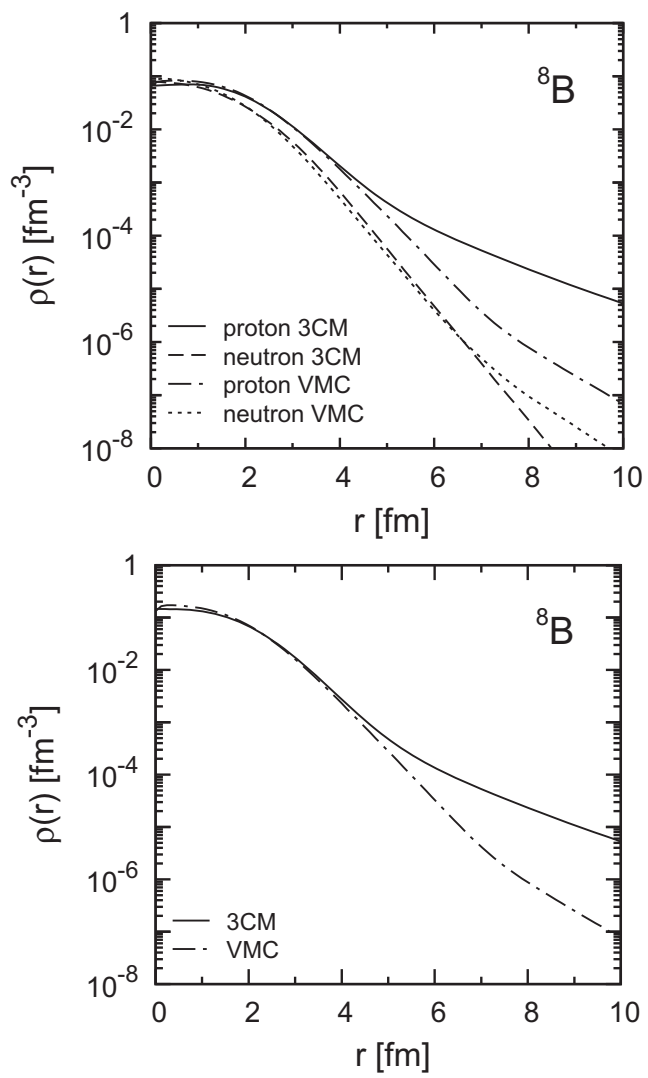

Figure 1. Point-proton (normalized to $Z=5$ ), point-neutron (normalized to $N=3$ ) (upper panel), and the total densities (bottom panel) of ${ }^{8} \mathrm{~B}$ (normalized to $A=8$ ) obtained in the VMC method [21] and in the 3CM [22].

al. [22]. Concerning the beryllium ${ }^{12,14} \mathrm{Be}$ isotopes, we apply the density distributions obtained within the generator coordinate method [23]. Additionally, for the ${ }^{12} \mathrm{Be}$ density we use the one obtained in the framework of the same VMC model. The VMC and $3 \mathrm{CM}$ densities of ${ }^{8} \mathrm{~B}$ are given in Fig. 1. It can be seen that they have been calculated with enough accuracy up to distances much larger than the nuclear radius. In both methods the total densities of ${ }^{8} \mathrm{~B}$ occur quite similar up to $r \sim 4 \mathrm{fm}$ and a difference between them is seen in their periphery. Due to the cluster-structure model of ${ }^{8} \mathrm{~B}$, where the proton is considered as a single cluster [22], the tail part of the pointproton distribution of ${ }^{8} \mathrm{~B}$ is significantly larger than that of the neutron one, causing considerable difference in the corresponding rms radii (see Ref. [7]). The ${ }^{14} \mathrm{Be}$ nucleus is investigated in the three-cluster GCM, involving several ${ }^{12} \mathrm{Be}+n+n$ configurations [23]. As can be seen from Fig. 2, the proton densities are very similar in ${ }^{12} \mathrm{Be}$ and ${ }^{14} \mathrm{Be}$ nuclei. On the contrary, neutron densities are quite different; whereas neutron density in ${ }^{12} \mathrm{Be}$ is nearly proportional to the proton density, the neutron contribution in ${ }^{14} \mathrm{Be}$ has a very long tail. This long-range neutron density is typical for neutron-rich halo nuclei and yields fairly large rms radii (value of $2.95 \mathrm{fm}$ obtained in GCM was reported in Ref. [23]).

The calculated within the hybrid model elastic scattering cross sections of ${ }^{8} \mathrm{~B}+{ }^{12} \mathrm{C}$ at energy $E=25.8 \mathrm{MeV}$ in 

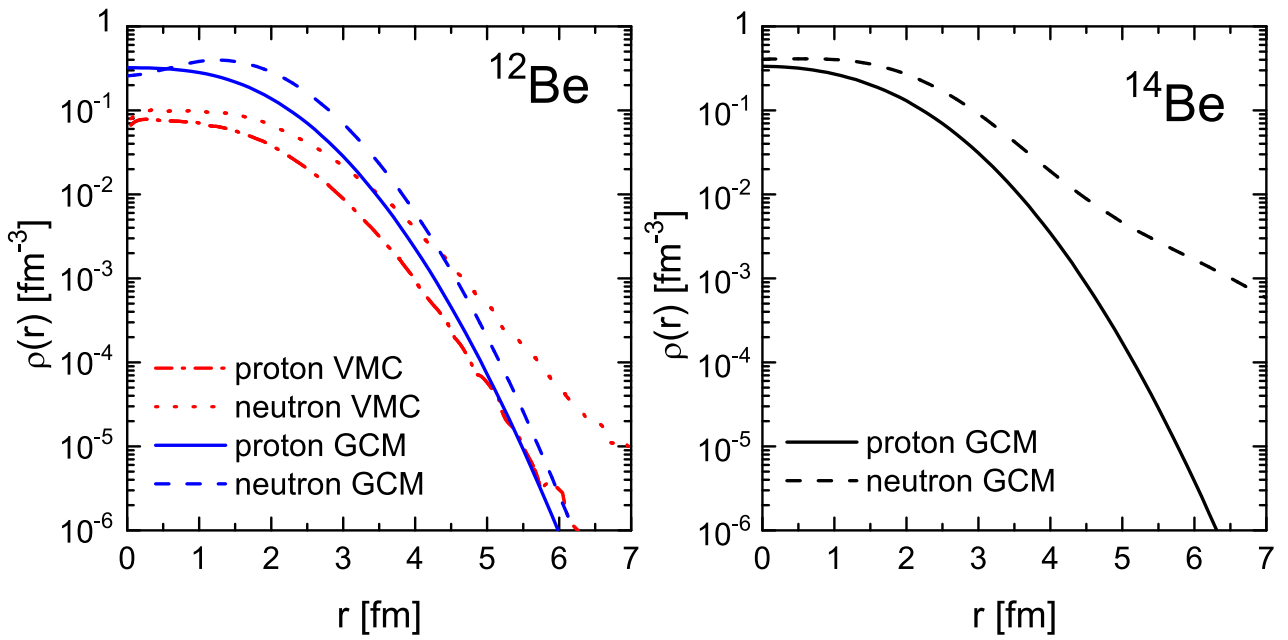

Figure 2. Point-proton and point-neutron densities of ${ }^{12} \mathrm{Be}$ (left panel) obtained in the VMC method [21] and in the GCM [23], and the same densities (right panel) of ${ }^{14} \mathrm{Be}$ obtained in the GCM [23].

the laboratory frame are given in Fig. 3 and compared with the experimental data [8]. It can be seen that in both cases of calculations with VMC or 3CM densities the results are in good agreement with the available data. The differential cross section obtained with VMC density demonstrates more developed diffractional picture. It would be desirable to measure the elastic scattering in the angular range beyond $55^{\circ}$, where the differences between the theoretical results start, in order to determine the advantage of using VMC or $3 \mathrm{CM}$ microscopic densities of ${ }^{8} \mathrm{~B}$. Complementary measurements at smaller steps of scattering angle would also allow one to observe some possible oscillations of the cross section. Our next step is to study ${ }^{8} \mathrm{~B}$ elastic scattering on a lead target at $170.3 \mathrm{MeV}$ incident energy. Figure 4 shows a fair agreement of our microscopic calculations with the experimental data for the cross section. Both VMC and 3CM densities used in the calculations are able to reproduce the data that are restricted in a range of small angles. Similarly to the case of ${ }^{8} \mathrm{~B}+{ }^{12} \mathrm{C}$ process, the reasonable agreement of our model with the data on ${ }^{8} \mathrm{~B}+{ }^{208} \mathrm{~Pb}$ elastic scattering is in favor of the very weak contribution from other reaction mechanisms, which is supported by the results from CDCC calculations $[8,10]$.

Next, we present in Fig. 5 our results for ${ }^{8} \mathrm{~B}+{ }^{58} \mathrm{Ni}$ elastic scattering cross sections at energies 20.7, 23.4, 25.3, 27.2, and 29.3 MeV using the VMC density. These results are obtained with $N_{R}$ and $N_{I}$ which reproduce in a best way the experimental cross sections at considered five energies. One can see that the results are in a good agreement with the data for all energies considered. It is well known that the couplings to non-elastic channels lead to polarization potentials that can considerably modify the bare potential calculated within the double folding formalism. Obviously, for more successful description of cross sections at low energies near Coulomb barrier an inclusion of polarization contributions due to virtual excitations and decay channels of the reactions is necessary to obtain unambiguously the OP renormalization parameters. The good fit obtained for the experimental angular distributions in

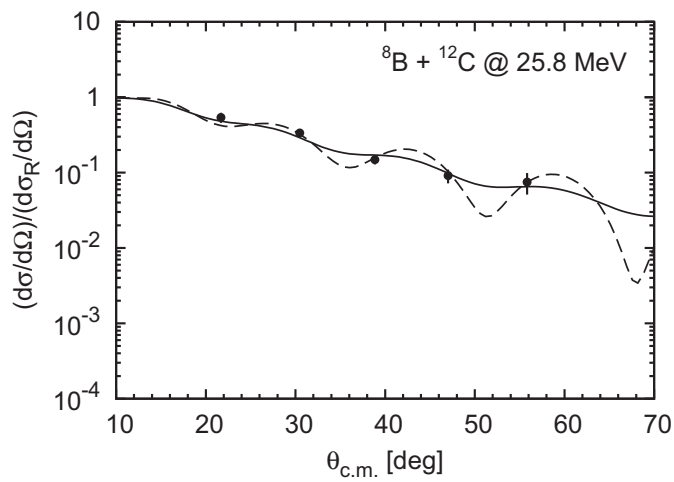

Figure 3. ${ }^{8} \mathrm{~B}+{ }^{12} \mathrm{C}$ elastic scattering cross sections at $E=25.8$ $\mathrm{MeV}$. Solid line: calculations with $3 \mathrm{CM}$ density of ${ }^{8} \mathrm{~B}$; dashed line: calculations with VMC density of ${ }^{8} \mathrm{~B}$. Experimental data are taken from Ref. [8].

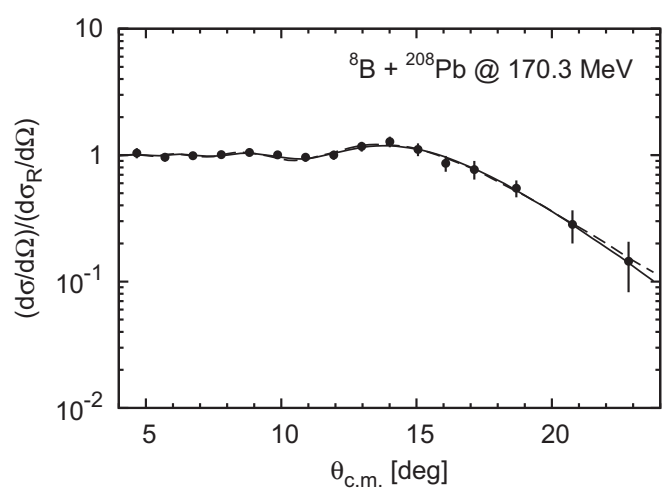

Figure 4. ${ }^{8} \mathrm{~B}+{ }^{208} \mathrm{~Pb}$ elastic scattering cross sections at $E=170.3$ $\mathrm{MeV}$. Solid line: calculations with $3 \mathrm{CM}$ density of ${ }^{8} \mathrm{~B}$; dashed line: calculations with VMC density of ${ }^{8} \mathrm{~B}$. Experimental data are taken from Ref. [10]. 


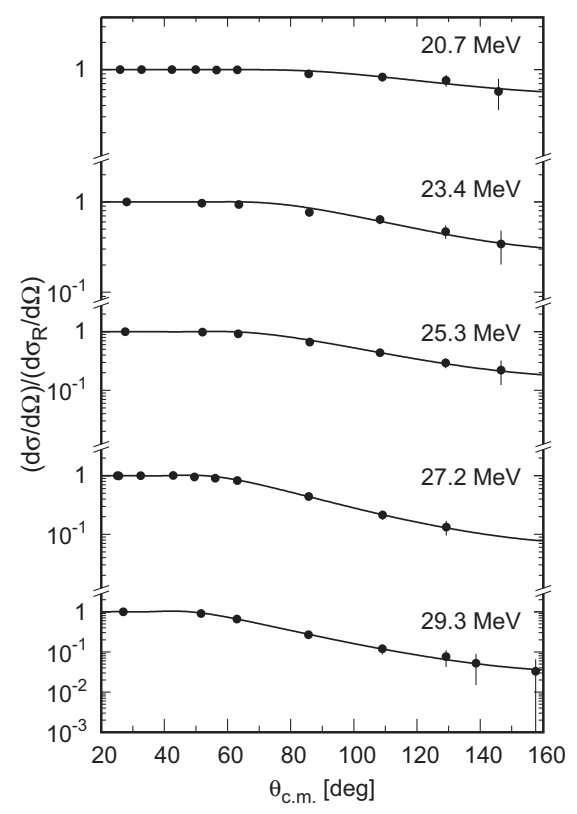

Figure 5. ${ }^{8} \mathrm{~B}+{ }^{58} \mathrm{Ni}$ elastic scattering cross sections at $E=$ 20.7, 23.4, 25.3, 27.2 and 29.3 MeV calculated using the VMC density of ${ }^{8} \mathrm{~B}$. Experimental data are taken from Ref. [9].

Ref. [9] with real and imaginary potentials of the WoodsSaxon type and our best fit to the same data using microscopic OP in this work lead to values of the predicted total reaction cross section $\sigma_{R}$ very close to each other (see [7]), the latter exhibiting a smooth increase with the energy increase.

Also, we give in Fig. 6, as an example (for $E=29.3$ $\mathrm{MeV}$ ), the comparison of the obtained real and imaginary parts of the OPs for both 3CM and VMC densities with the corresponding parts of the fitted Woods-Saxon (WS) potential used in [9]. The values of our parameters $N_{R}$ and $N_{I}$ are indicated in the figure. Here we mention that at such energies the surface part of the ImOP plays a decisive role on the behavior of the elastic cross sections. One can see that the use of the VMC density leads to a very good agreement of the imaginary part of our OP with the imaginary part of the fitted WS OP in the surface region. Also, the slope of the real part of OP obtained with the VMC density in this region $(8<r<10 \mathrm{fm})$ is similar to that of the real part of WS OP. There exist some differences in the surface region for the real and imaginary parts of the OP obtained with the 3CM density and the corresponding parts of the WS OP.

The role of the density distribution of the ${ }^{12} \mathrm{C}$ target on the elastic ${ }^{14} \mathrm{Be}+{ }^{12} \mathrm{C}$ scattering cross section at incident energy $56 \mathrm{MeV} /$ nucleon is illustrated in Fig. 7. The corresponding OPs are calculated with GCM density of ${ }^{14} \mathrm{Be}$ and density of ${ }^{12} \mathrm{C}$ taken in the form of i) symmetrized Fermi (SF) function and ii) modified SF function [24], in which a derivative term simulating the effect of the nuclear surface is added to the traditional SF term. It can be seen from Fig. 7 that the use of the modified SF density of ${ }^{12} \mathrm{C}$ leads to a left-shift correction of the minimum that is lo-

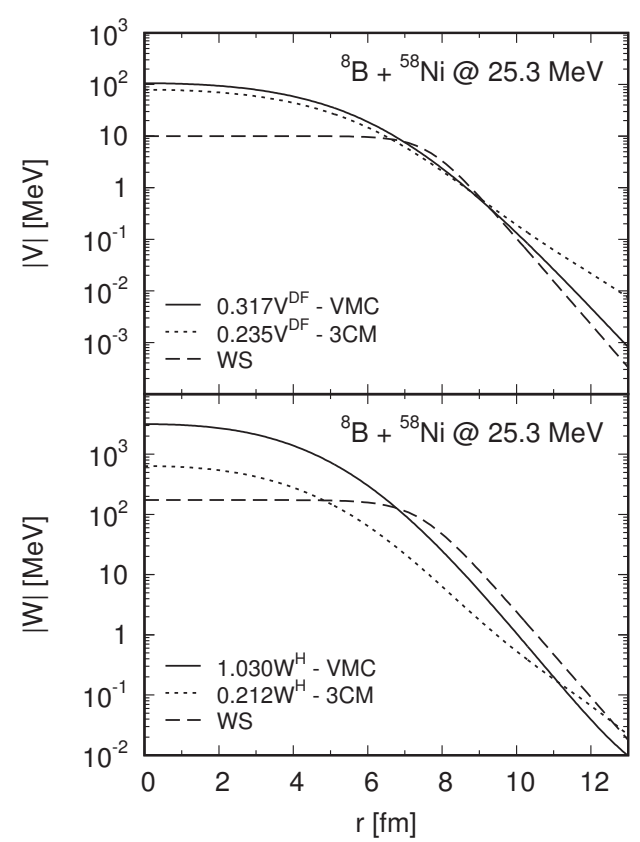

Figure 6. The absolute values of the real $N_{R} V^{D F}$ and imaginary $N_{I} W^{H}$ parts of the calculated optical potentials for the ${ }^{8} \mathrm{~B}+{ }^{58} \mathrm{Ni}$ elastic scattering at $E=29.3 \mathrm{MeV}$ obtained using the VMC and $3 \mathrm{CM}$ densities of ${ }^{8} \mathrm{~B}$ in comparison with those of the WS potential from Ref. [9].

cated at around $3^{\circ}$. However, this minimum still stays deep in comparison with the experimental data. Obviously, the account for the inelastic scattering to the first $2^{+}$excited state in ${ }^{12} \mathrm{C}$ in the quasielastic process would provide a better agreement with the data.

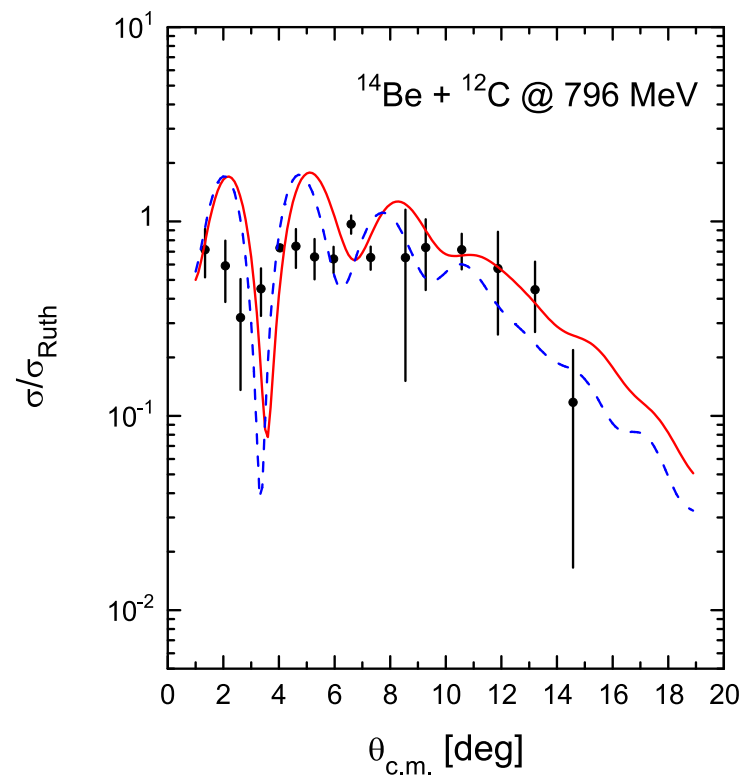

Figure 7. ${ }^{14} \mathrm{Be}+{ }^{12} \mathrm{C}$ elastic scattering cross sections at $E=56$ $\mathrm{MeV} /$ nucleon. Solid line: calculations with SF function density of ${ }^{12} \mathrm{C}$; dashed line: calculations with modified SF density of ${ }^{12} \mathrm{C}$. Experimental data are taken from Ref. [25]. 


\section{Breakup reactions of ${ }^{8} \mathrm{~B}$}

In this section we consider the characteristics of breakup processes of the ${ }^{8} \mathrm{~B}$ nucleus on the example of the stripping reaction cross sections and the momentum distributions of the fragments. We use a simple model in which ${ }^{8} \mathrm{~B}$ consists of a core of ${ }^{7} \mathrm{Be}$ and a halo of a single proton (see, e.g., Refs. [3]). In this model the density of ${ }^{7} \mathrm{Be}$ has to be known. We use that one obtained from the calculations performed by means of the $3 \mathrm{CM}$ density of ${ }^{8} \mathrm{~B}$ [22].

For calculations of breakup cross sections and momentum distributions of fragments in the ${ }^{7} \mathrm{Be}+p$ breakup model within the eikonal formalism (see, e.g. Ref. [26]), one needs the expressions of the $S$-matrix (as a function of the impact parameter $b$ ):

$$
S(b)=\exp \left[-\frac{i}{\hbar v} \int_{-\infty}^{\infty} U^{(b)}\left(\sqrt{b^{2}+z^{2}}\right) d z\right],
$$

where

$$
U^{(b)}=V+i W
$$

is the OP of the breakup of ${ }^{8} \mathrm{~B}$ in its collision with nuclear targets within the ${ }^{7} \mathrm{Be}+p$ cluster model. The longitudinal momentum distribution of ${ }^{7} \mathrm{Be}$ fragments produced in the breakup of ${ }^{8} \mathrm{~B}$ in the case of the stripping reaction (when the proton leaves the elastic channel) is (more details can be found in Ref. [7])

$$
\begin{aligned}
\left(\frac{d \sigma}{d k_{L}}\right)_{s t r} & =\frac{1}{3} \int_{0}^{\infty} b_{p} d b_{p}\left[1-\left|S_{p}\left(b_{p}\right)\right|^{2}\right] \\
& \times \int \rho d \rho d \varphi_{\rho}\left|S_{c}\left(b_{c}\right)\right|^{2} \sum_{m=0, \pm 1} F_{m}(\rho) .
\end{aligned}
$$

The wave function of the relative motion of the proton and ${ }^{7} \mathrm{Be}$ clusters in ${ }^{8} \mathrm{~B}$ is obtained by solving the Schrödinger equation with the Woods-Saxon potential for a particle with a reduced mass of two clusters. The parameters of the WS potentials are obtained by a fitting procedure, namely, to reach the proton separation energy $S_{p}=137 \mathrm{KeV}$. However, this procedure could provide several sets of potential parameters that satisfy the above condition. They are close to each other leading, at the same time, to different valence proton rms radii. Therefore, in order to understand better the observed widths of the longitudinal momentum distributions of ${ }^{7} \mathrm{Be}$ fragments formed in the breakup of ${ }^{8} \mathrm{~B}$ and measured at different targets and energies, we consider three cases. The values of WS potential parameters and corresponding rms radii of the cluster formation for $1 p$ state in which the valence proton in ${ }^{8} \mathrm{~B}$ is mainly bound (see Refs. $[6,26]$ ) are listed in Table 1.

The stripping cross sections for reactions ${ }^{8} \mathrm{~B}+{ }^{9} \mathrm{Be}$, ${ }^{8} \mathrm{~B}+{ }^{12} \mathrm{C}$, and ${ }^{8} \mathrm{~B}+{ }^{197} \mathrm{Au}$ are calculated from Eq. (7). The obtained results are illustrated in Fig. 8. The blue dotted, black solid, and red dashed curves in the figures correspond to the three sets of WS parameters given in Table 1 . Here we note that due to the arbitrary units of the measured cross sections of the considered processes it was not necessary to renormalize the depths of our OPs of the fragmentstarget nuclei interactions. It is worth to note the relevance
Table 1. The parameters $V_{0}$ (in $\mathrm{MeV}$ ), $R$ (in $\mathrm{fm}$ ), $a$ (in $\mathrm{fm}$ ) of the Woods-Saxon potentials and the rms radii of the cluster wave function (in fm) obtained by using of the $3 \mathrm{CM}$ density of ${ }^{7} \mathrm{Be}$ for three cases (see the text).

\begin{tabular}{llll}
\hline$V_{0}$ & $R$ & $a$ & rms radii \\
\hline 38.22 & 2.70 & 0.55 & 4.51 \\
38.70 & 2.50 & 0.20 & 5.08 \\
38.77 & 2.48 & 0.50 & 6.24 \\
\hline
\end{tabular}

between the rms radii of the wave function of the ${ }^{7} \mathrm{Be}$ $p$ relative motion and the obtained FWHMs for the considered three cases. Due to the uncertainty principle the widths become smaller with the increase of the distance between the two clusters. We note the good agreement with the experimental data from light and heavy breakup targets. It can be seen from Fig. 8 that the best agreement with the experimental data for the parallel momentum distributions of ${ }^{7} \mathrm{Be}$ fragments in a breakup reaction of ${ }^{8} \mathrm{~B}$ on a ${ }^{9} \mathrm{Be}$ target at $41 \mathrm{MeV} /$ nucleon and on a ${ }^{12} \mathrm{C}$ target at $36 \mathrm{MeV} /$ nucleon is achieved when the relative ${ }^{7} \mathrm{Be}$-proton distance is $5.08 \mathrm{fm}$ or $4.51 \mathrm{fm}$, respectively, while in the case of ${ }^{8} \mathrm{~B}$ breakup on a ${ }^{197} \mathrm{Au}$ target at 41 $\mathrm{MeV} /$ nucleon shown in the same figure a larger distance $(6.24 \mathrm{fm})$ is needed to get better coincidence with the data. The values of the theoretical FWHMs in this case are 72.07 $\mathrm{MeV} / \mathrm{c}$ when describing the stripping mechanism of the ${ }^{8} \mathrm{~B}$ breakup on the ${ }^{9} \mathrm{Be}$ target, $108.71 \mathrm{MeV} / \mathrm{c}$ for the breakup on ${ }^{12} \mathrm{C}$ target, and 54.86 in the case of ${ }^{197} \mathrm{Au}$ target. These values are close to the experimentally measured widths and are within the range found in other theoretical analyses.

\section{Conclusions}

In the present work (see also [7]) we performed microscopic calculations of the optical potentials and cross sections of elastic scattering ${ }^{8} \mathrm{~B}+{ }^{12} \mathrm{C}$ at $25.8 \mathrm{MeV},{ }^{8} \mathrm{~B}+{ }^{58} \mathrm{Ni}$ at 20.7, 23.4, 25.3, 27.2, and $29.3 \mathrm{MeV},{ }^{8} \mathrm{~B}+{ }^{208} \mathrm{~Pb}$ at 170.3 $\mathrm{MeV}$, and ${ }^{12,14} \mathrm{Be}+{ }^{12} \mathrm{C}$ at $56 \mathrm{MeV} /$ nucleon in comparison with the available experimental data. The direct and exchange isoscalar and isovector parts of the real OP $\left(V^{D F}\right)$ were calculated microscopically using the double-folding procedure and density dependent M3Y (CDM3Y6-type) effective interaction based on the Paris NN potential. The imaginary part of the OP $\left(W^{H}\right)$ was calculated as a folding integral that corresponds to the one in a phase of HEA. Two model microscopic densities of protons and neutrons in ${ }^{8} \mathrm{~B}$ were used in the calculations: the density calculated within the VMC model [21] and from the three-cluster model [22]. The VMC model densities of ${ }^{12} \mathrm{Be}$ and GCM densities of ${ }^{12,14}$ Be nuclei [23] have been explored.

We have tested our microscopic model studying the role of the breakup mechanism to analyze properly the whole picture of ${ }^{8} \mathrm{~B}$ scattering. For this purpose, we use another folding approach to consider the ${ }^{8} \mathrm{~B}$ breakup by means of the simple ${ }^{7} \mathrm{Be}+p$ cluster model for the structure of ${ }^{8} \mathrm{~B}$. We calculate in HEA the ImOP of the interaction of ${ }^{7} \mathrm{Be}$ with the target, as well as the $p+$ target interaction. Using them the corresponding $S$-matrices for the core and 

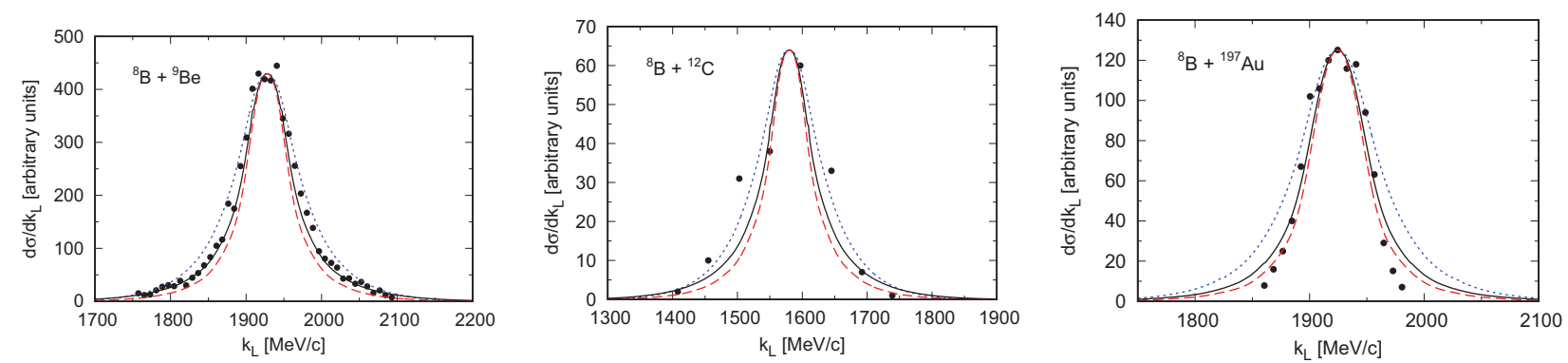

Figure 8. Cross sections of stripping reactions in ${ }^{8} \mathrm{~B}+{ }^{9} \mathrm{Be}$ scattering at $E=41 \mathrm{MeV} /$ nucleon, ${ }^{8} \mathrm{~B}+{ }^{12} \mathrm{C}$ scattering at $E=36 \mathrm{MeV} / \mathrm{nucleon}$, and ${ }^{8} \mathrm{~B}+{ }^{197} \mathrm{Au}$ scattering at $E=41 \mathrm{MeV} /$ nucleon. Experimental data are taken from Refs. [4], [5], and [4], respectively.

proton within the eikonal formalism are obtained. The latter are used to get results for the longitudinal momentum distributions of ${ }^{7} \mathrm{Be}$ fragments produced in the breakup of ${ }^{8} \mathrm{~B}$ on different targets. This includes the breakup reactions of ${ }^{8} \mathrm{~B}$ on ${ }^{9} \mathrm{Be}$ and ${ }^{197} \mathrm{Au}$ at $E=41 \mathrm{MeV} /$ nucleon and ${ }^{8} \mathrm{~B}$ on ${ }^{12} \mathrm{C}$ at $E=36 \mathrm{MeV} /$ nucleon, for which a good agreement of our calculations for the stripping reaction cross sections with the available experimental data were obtained. The theoretical widths are close to the empirical ones.

The authors are grateful to S. C. Pieper for providing with the density distributions of ${ }^{8} \mathrm{~B}$ nucleus calculated within the VMC method, to P. Descouvemont for the density distributions of ${ }^{12,14} \mathrm{Be}$ nuclei obtained within the GCM, and to S. L. Jin for the experimental longitudinal momentum distributions of ${ }^{7} \mathrm{Be}$ fragments from the breakup of ${ }^{8} \mathrm{~B}$ on a carbon target. Three of the authors (V.K.L., E.V.Z. and K.V.L.) thank the Russian Foundation for Basic Research (Grant No. 17-52-18057 bolg-a) for the partial support. Four of the authors (D.N.K., A.N.A., M.K.G. and K.S.) are grateful for the support of the Bulgarian Science Fund under Contracts No. DFNI-T02/19 and No. DNTS/Russia 01/3, and one of them (D.N.K.) under Contract No. DFNI-E02/6.

\section{References}

[1] B. Davids et al., Phys. Rev. Lett. 81, 2209 (1998)

[2] L. Trache et al., Phys. Rev. C 67, 062801 (2003)

[3] W. Schwab et al., Z. Phys. A 350, 283 (1995)

[4] J. H. Kelley et al., Phys. Rev. Lett. 77, 5020 (1996)

[5] S. L. Jin et al., Phys. Rev. C 91, 054617 (2015)

[6] H. Esbensen, Phys. Rev. C 53, 2007 (1996)

[7] V. K. Lukyanov, D. N. Kadrev, E. V. Zemlyanaya, K. V. Lukyanov, A. N. Antonov, M. K. Gaidarov, and K. Spasova, Eur. Phys. J. A 53, 31 (2017)
[8] A. Barioni et al., Phys. Rev. C 84, 014603 (2011)

[9] E. F. Aguilera et al., Phys. Rev. C 79, 021601(R) (2009)

[10] Y. Y. Yang et al., Phys. Rev. C 87, 044613 (2013)

[11] K. V. Lukyanov et al., Eur. Phys. J. A 33, 389 (2007)

[12] V. K. Lukyanov et al., Phys. Rev. C 80, 024609 (2009)

[13] V. K. Lukyanov et al., Phys. Rev. C 82, 024604 (2010)

[14] V. K. Lukyanov et al., Phys. Rev. C 88, 034612 (2013)

[15] V. K. Lukyanov et al., Phys. Rev. C 91, 034606 (2015)

[16] K. V. Lukyanov, E. V. Zemlyanaya, and V. K. Lukyanov, Phys. At. Nucl. 69, 240 (2006)

[17] D. T. Khoa and G. R. Satchler, Nucl. Phys. A 668, 3 (2000)

[18] R. J. Glauber, Lectures in Theoretical Physics (Interscience, New York, 1959) 315

[19] A. G. Sitenko, Ukr. Fiz. J. 4, 152 (1959)

[20] P. Shukla, Phys. Rev. C 67, 054607 (2003)

[21] S. C. Pieper, private communication.

[22] K. Varga, Y. Suzuki, and I. Tanihata, Phys. Rev. C 52, 3013 (1995)

[23] P. Descouvemont, Phys. Rev. C 52, 704 (1995)

[24] V. V. Burov, D. N. Kadrev, V. K. Lukyanov, and Yu. S. Pol', Phys. At. Nucl. 61, 525 (1998)

[25] M. Zahar et al., Phys. Rev. C 49, 1540 (1994)

[26] K. Hencken, G. Bertsch, and H. Esbensen, Phys. Rev. C 54, 3043 (1996) 